\title{
Liver cancer incidence and mortality in China: Temporal trends and projections to 2030
}

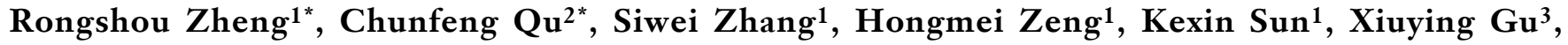 \\ Changfa Xia ${ }^{1}$, Zhixun Yang ${ }^{1}$, He Li ${ }^{1}$, Wenqiang Wei ${ }^{1}$, Wanqing Chen ${ }^{4}$, Jie $\mathrm{He}^{5}$
}

${ }^{1}$ Office for Cancer Registry, National Cancer Center/National Clinical Research Center for Cancer/Cancer Hospital, Chinese Academy of Medical Science and Peking Union Medical College, Beijing 100021, China; ${ }^{2}$ State Key Laboratory of Molecular Oncology, National Cancer Center/National Clinical Research Center for Cancer/Cancer Hospital, Chinese Academy of Medical Sciences and Peking Union Medical College, Beijing 100021, China; ${ }^{3}$ Cancer Research Institute, Affiliated Tumor Hospital, Xinjiang Medical University, Urumqi 830011, China; ${ }^{4}$ Office of Cancer Screening, National Cancer Center/National Clinical Research Center for Cancer/Cancer Hospital, Chinese Academy of Medical Science and Peking Union Medical College, Beijing 100021, China; ${ }^{5}$ Department of Thoracic Surgery, National Cancer Center/National Clinical Research Center for Cancer/Cancer Hospital, Chinese Academy of Medical Science and Peking Union Medical College, Beijing 100021, China

*These authors contributed equally for this work.

Correspondence to: Prof. Wanqing Chen. Office of Cancer Screening, National Cancer Center/National Clinical Research Center for Cancer/Cancer Hospital, Chinese Academy of Medical Science and Peking Union Medical College, No. 17 Panjiayuan South Lane, Chaoyang District, Beijing 100021, China. Email: chenwq@cicams.ac.cn; Prof. Jie He. Department of Thoracic Surgery, National Cancer Center/National Clinical Research Center for Cancer/Cancer Hospital, Chinese Academy of Medical Science and Peking Union Medical College, No. 17 Panjiayuan South Lane, Chaoyang District, Beijing 100021, China. Email: hejie@cicams.ac.cn.

\begin{abstract}
Objective: Liver cancer is one of the most common cancers and major cause of cancer deaths in China, which accounts for over $50 \%$ of new cases and deaths worldwide. The systematic liver cancer statistics including of projection through 2030 could provide valuable information for prevention and control strategies in China, and experience for other countries.

Methods: The burden of liver cancer in China in 2014 was estimated using 339 cancer registries' data selected from Chinese National Cancer Center (NCC). Incident cases of 22 cancer registries were applied for temporal trends from 2000 to 2014. The burden of liver cancer through 2030 was projected using age-period-cohort model.

Results: About 364,800 new cases of liver cancer (268,900 males and 95,900 females) occurred in China, and about 318,800 liver cancer deaths (233,500 males and 85,300 females) in 2014. Western regions of China had the highest incidence and mortality rates. Incidence and mortality rates decreased by about $2.3 \%$ and $2.6 \%$ per year during the period of 2000-2014, respectively, and would decrease by more than 44\% between 2014 and 2030 in China. The young generation, particularly for those aged under 40 years, showed a faster down trend.

Conclusions: Based on the analysis, incidence and mortality rates of liver cancer are expected to decrease through 2030, but the burden of liver cancer is still serious in China, especially in rural and western areas. Most cases of liver cancer in China can be prevented through vaccination and more prevention efforts should be focused on high risk groups.
\end{abstract}

Keywords: Liver cancer burden; temporal trends; prediction; cancer registry; China

Submitted Oct 26, 2018. Accepted for publication Nov 28, 2018.

doi: 10.21147/j.issn.1000-9604.2018.06.01

View this article at: https://doi.org/10.21147/j.issn.1000-9604.2018.06.01 


\section{Introduction}

Liver cancer is one of the most common cancers diagnosed and causes of cancer death worldwide. It was estimated of 782,451 new liver cancer cases and nearly 746,000 deaths in 2012 (1). Liver cancer comprises two major histological types of heterogeneous malignant diseases: hepatocellular carcinoma (HCC) and intrahepatic cholangiocarcinoma (ICC) (2). Generally, $70 \%-85 \%$ of liver cancer cases are HCC and $10 \%-20 \%$ are ICC histologically $(2,3)$. Approximately $80 \%$ of liver cancer cases were attributed to chronic infection of hepatitis $\mathrm{B}$ virus (HBV) and hepatitis $\mathrm{C}$ virus (HCV) (4). While other factors, including dietary aflatoxin, algal hepatotoxins in drinking water, betel nut chewing, diabetes mellitus, non-alcoholic fatty liver disease, alcohol consumption and tobacco use, have been reported as potential risk factors of liver cancer $(3,5)$. Primary prevention is effective through behaviours intervention and vaccination $(3,6,7)$. Studies show that individuals with $\mathrm{HBV}$ positive could benefit from analogue $(8,9)$.

The age-standardized incidence rates of liver cancer have been declined in the most of Asia countries, including China, but increased in the Europeans and North Americans during the past decades (10-12). In China, $83.9 \%-92.3 \%$ liver cancers are HCC $(13,14)$. Chronic $\mathrm{HBV}$ infection acquired through vertical or horizontal infection in early life is the major cause of liver cancer in China, countries of East and South-East Asia (except Japan), and Middle/Western Africa (5). After HBV vaccine was available in early 1980s, the prevalence of HBV infection kept decreasing in beneficiaries, especially in young generation $(15,16)$. Previous study reported that the age-standardized incidence rates of liver cancer decreased by $1.8 \%$ annually from 2000 to 2011 (17). The statewide $\mathrm{HBV}$ vaccination has been incorporated into neonatal immunization program in China since 2002 (18). Reduction of liver cancer incidence in some areas was not fully attributed to the vaccination program (7). Therefore, the changing in liver cancer incidence in China and the other countries might reflect different etiologies and the strength of different risk factors.

Liver cancer incidence in China is higher compared with other countries worldwide. China contains about $19 \%$ of the world population but accounts for over $50 \%$ of all newly diagnosed liver cancer cases and deaths (19-21). In this study, we provide the estimated numbers of new liver cancer cases and deaths in 2014 in China nationally based on the most current population-based data, and the temporal trends during the past decades and predictions to
2030, which could provide some valuable information for the other countries to develop better intervention strategies/approaches to control the disease.

\section{Materials and methods}

\section{Data source and quality control}

All data were retrieved from the Chinese National Cancer Center (NCC), which takes responsibility for collecting, evaluating the data from local population-based cancer registries located in different provinces of China and publishing national cancer statistics. Local cancer registries submit data to NCC and quality control is conducted based on the criteria of Chinese Guideline for Cancer Registration and Cancer Incidence in Five Continents (CI5). Briefly, the completeness, comparability and validity of the data are evaluated by indicators, such as mortality to incidence $(M / I)$ ratio, the percentage of cases morphologically verified (MV\%), the percentage of death certificate-only cases (DCO\%), and stability of cancer trends over years. The International Classification of Diseases for Oncology, 3rd edition (ICD-O-3) and the International Statistical Classification of Diseases and Related Health Problems 10th Revision (ICD-10) are both applied for coding. Qualified cancer registries' data were included in this study (Supplementary Figure S1). Detailed information was provided in the Supplementary materials.

\section{Statistical analysis}

To analyze the incidence and mortality of liver cancer in 2014, we extracted the data coded as C22 of ICD-10 from the data source. Crude incidence and mortality rates were calculated by area (urban/rural), gender (male/female) and age groups $(0,1-4,5$ to 80 by 5 years, $85+)$. To calculate the estimated numbers of new cancer cases and deaths in 2014 , the numbers in each stratum were computed using age-specific incidence or mortality rates from 339 cancer registries multiplied by corresponding estimated agespecific population in each stratum and then added up. Each of the cancer registries provided population data in the respective areas to the NCC.

The temporal trends in incidence and mortality of liver cancer from 2000 to 2014 were examined by fitting Joinpoint Regression Program (Version 4.1.1, https:// surveillance.cancer.gov/joinpoint/) to the age-standardized rates using the data from 22 cancer registries. Those 22 cancer registries with qualified and continued data were established since 1990 s, covering about $3.34 \%$ of China's 
national population. To reduce the possibility of reporting spurious changes in trends over the period, models were restricted to a maximum of 2 joinpoints (3-line segments) (17). Trends were expressed as an annual percent change (APC) and the $Z$ test was used to assess whether the change was statistically different from zero.

We age-standardized the incidence and mortality rates per 100,000 person-years using the world Segi's standard population (22). For each trend by the analysis, we computed the estimated APC by fitting a regression line to the natural logarithm of the rates using calendar year as a regression variable for each 5 -year age group (23). To project the number of new cases or deaths, we multiplied the projected rates from the logarithmic Poisson regression model by the projected population from the National Bureau of Statistics of China (http://data.stats.gov.cn/). We also apportioned predicted liver cancer cases and deaths into the contribution from the change of risk and change of population, including the population size and age structure according to methods described by Moller et al (24).

\section{Results}

Liver cancer incidence and mortality in China, 2014

We estimated about 364,800 new liver cancer cases
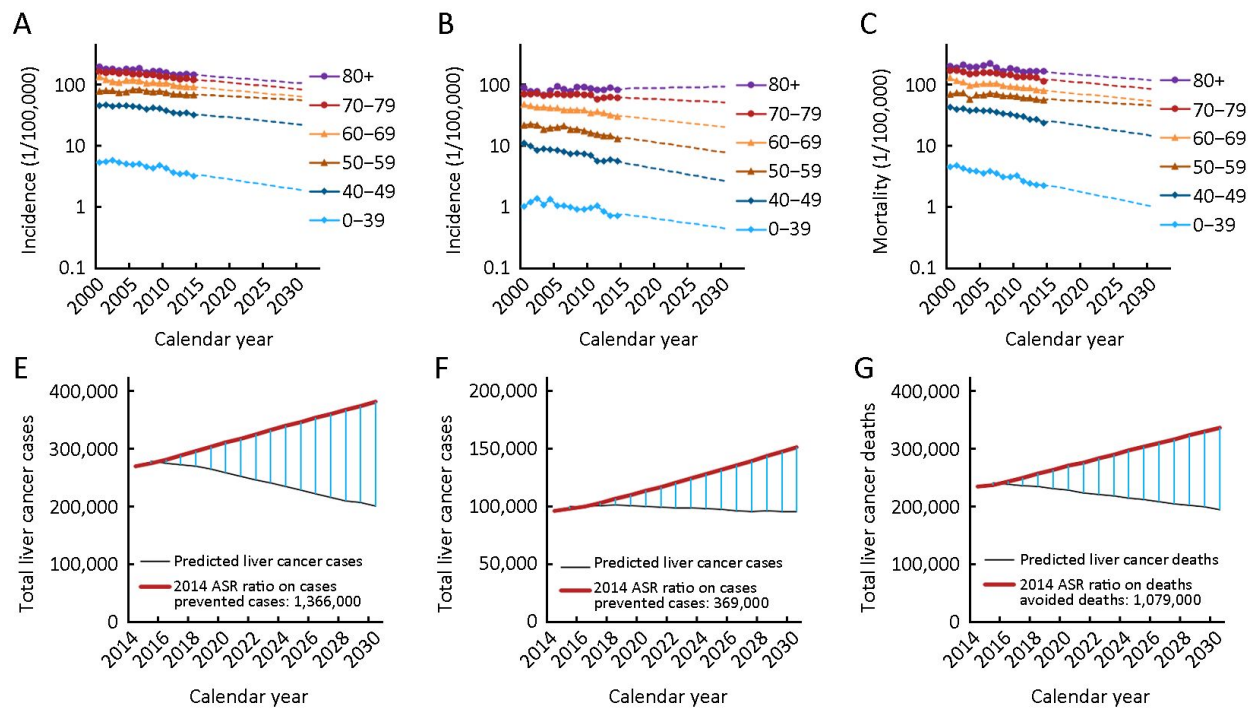

C

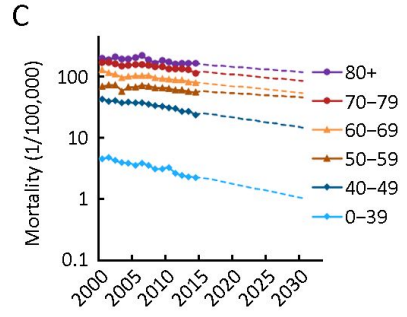

Calendar year

Calendar year

(268,900 males and 95,900 females) and about 318,800 liver cancer deaths (233,500 males and 85,300 females) in China in 2014. The crude and age-standardized incidence rates were 26.67 and 17.81 per 100,000 , respectively. The crude and age-standardized mortality rate was 23.31 and 15.29 per 100,000 , respectively. The incidence and mortality rates were higher in rural areas than in urban areas both in males and in females. The less-developed western areas of China showed the highest incidence and mortality rates, with followed by middle and eastern areas (Table 1).

\section{Trends in liver cancer incidence and mortality, 2000-2014}

Between 2000 and 2014, the age-standardized incidence rates of liver cancer decreased by about $2.2 \%$ per year in males and $2.5 \%$ per year in females (Figure 1, Table 2). Analysis of age-specific incidence rates showed that the decrease was mainly observed in the younger age groups $(<50$ years), particularly in those below 40 years with the decrease about $3.9 \%$ in males and $3.4 \%$ in females (Table 2). The decreasing trends were more obvious in rural areas. It decreased by about $4.0 \%$ of $<40$ years and $3.7 \%$ of $40-49$ age groups for males in rural areas compared to the urban areas $(-3.5 \%$ and $-2.2 \%$, respectively). In rural females, it decreased by about $5.3 \%$ of $<40$ years and $5.7 \%$ of $40-49$

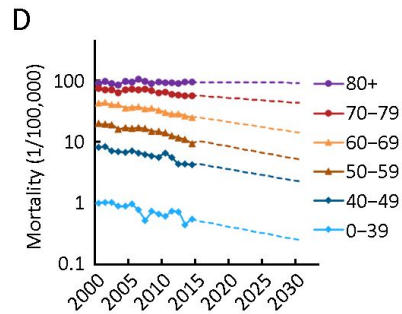

Calendar year

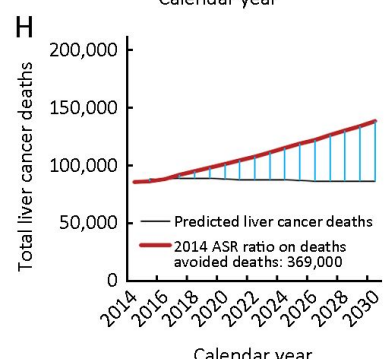

Figure 1 Trends for age-specific incidence and mortality rates during 2000-2014 and predicted from 2015 to 2030 , and the total prevented liver cancer cases and avoided deaths between the top rate in 2014 and 2030 in China. (A) Male incidence; (B) Female incidence; (C) Male mortality; (D) Female mortality; (E) Male cases; (F) Female cases; (G) Male deaths; (H) Female deaths. Right panel: black line represents predicted cancer cases or deaths from 2014 to 2030; red line represents estimated numbers of total liver cancer cases or deaths by applying 2014 age-specific incidence or mortality rate. 
Table 1 Estimated liver cancer incidence and mortality in China, 2014

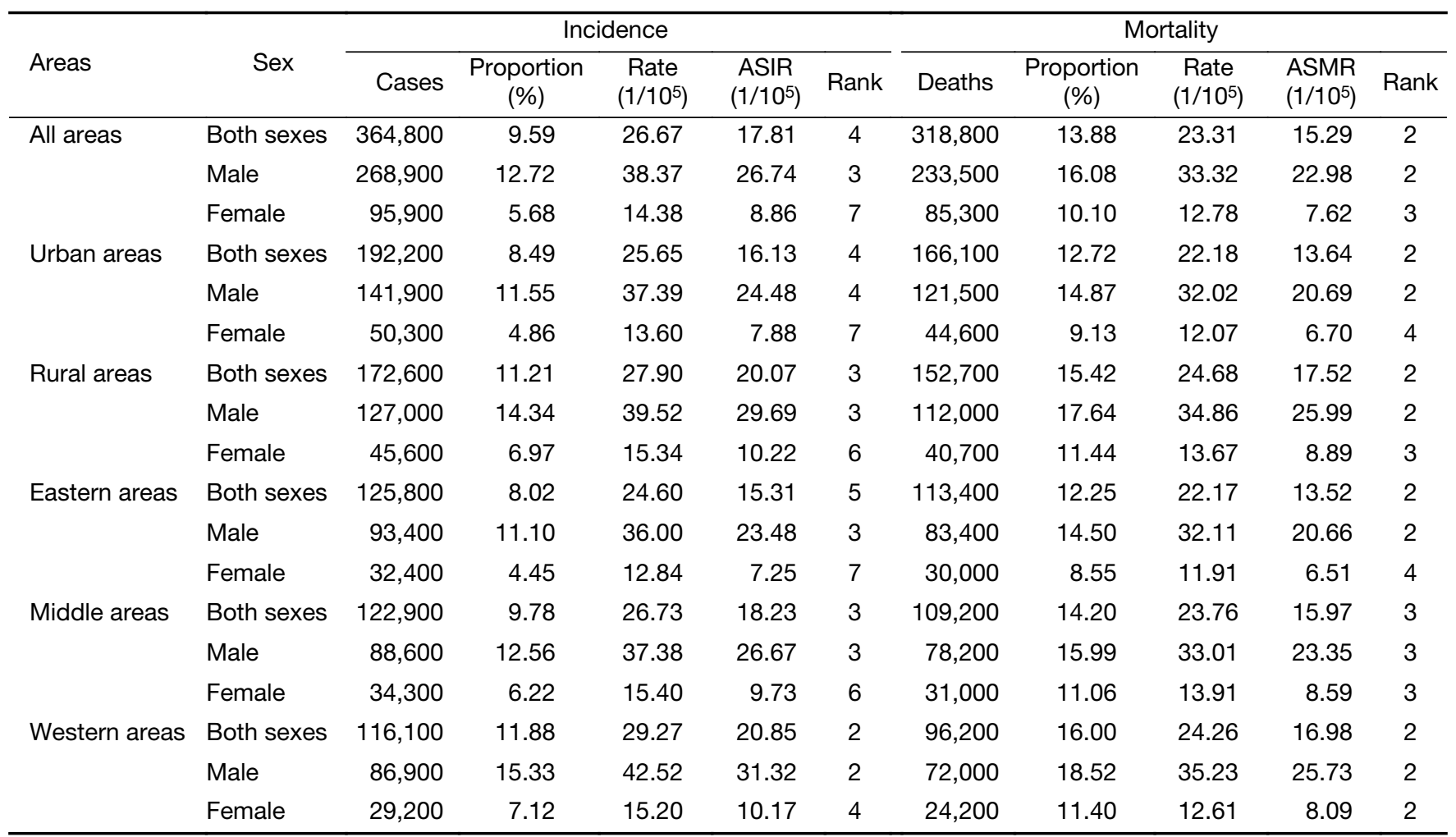

ASIR, age-standardized incidence rate using world Segi's standard population; ASMR, age-standardized mortality rate using world Segi's standard population.

Table 2 Trends for age-specific liver cancer incidence and mortality rates by area and gender in China, 2000 to 2014 (\%)

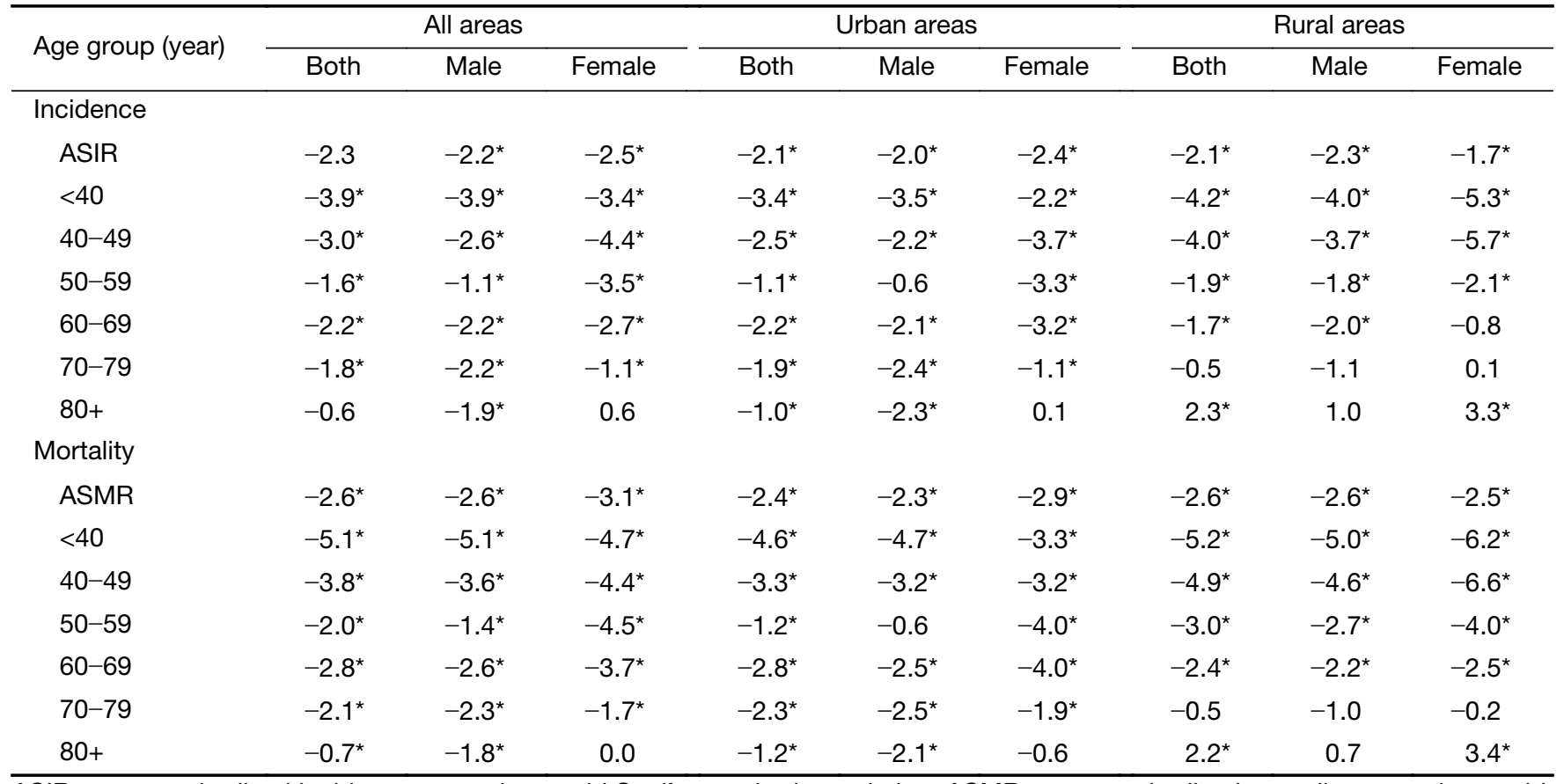

ASIR, age-standardized incidence rate using world Segi's standard population; ASMR, age-standardized mortality rate using world Segi's standard population; * average annual percent change during 2000 to 2014 is significantly different from zero $(P<0.05)$. 
age groups compared to the urban females $(-2.2 \%$ and $-3.7 \%$, respectively). Notably, in males of 50-59 age group, the incident rate was stable in urban areas, but it decreased in rural areas $(-1.8 \%)$. In females of $60-69$ age group, the incidence showed decrease in urban areas $(-3.2 \%, \mathrm{P}<0.05)$, but it was stable in rural areas. In the groups of $>70$ years, the incidence seemed stable and even increased in both rural males and rural females, different from the decline trends in urban areas (Table 2).

The long-term trends in liver cancer mortality rates were also analyzed by gender and age groups during 2000-2014. It showed the same trends as the incidence rates (Figure 1, Table 2). The age-standardized mortality rates decreased by about $2.6 \%$ per year in males and $3.1 \%$ per year in females. Also, the decreases were mainly observed in the age groups of $<50$ years, particularly in the group of $<40$ years. Also, the decrease was prominent in the rural areas compared to the urban areas. Likewise, in urban males of 50-59 age group, the mortality rate was stable, but it decreased by about $2.7 \%$ per year in rural areas (Table 2). Further analysis showed that a steady downward trend of incidence and mortality rates for all the age groups in male and in female happened in more recent birth cohorts, both in urban and in rural areas (Supplementary Figure S2). More details about the Joinpoint analysis are provided in Supplementary Table S1, S2.

\section{Projections of numbers of new cases and deatbs}

The overview of the projections of the future trend in liver cancer incidence, mortality and burden through 2030 by sex in China is presented in Table 3. The incidence and mortality rates for calendar years 2000 to 2014 and predicted trends from 2015 to 2030 are shown in Figure 1.
It is apparent from this graph that the forecast incidence and mortality rates will continue to decrease for most age groups. The age-standardized incidence and mortality rate decreased by more than $44 \%$ between 2014 and 2030. The number of new liver cancer incident cases decreased by about $18.90 \%$ with changing age-specific incidence rates (or can be explained by changes due to risk) contributing $-64.56 \%$, changing age structures contributing $40.67 \%$, and population growth contributing $5.00 \%$ to the overall decrease. The number of liver cancer deaths decreased by about $11.95 \%$ with changing age-specific mortality rates contributing $-60.99 \%$, changing age structures contributing $44.05 \%$, and population growth contributing $5.00 \%$ to the overall decrease (Table 3 ). The estimated number of predicted cancer cases and deaths from 2014 to 2030 due to the declines in rates since 2014 is showed in Figure 1 . We predict that about 1,762,000 liver cancer cases could be prevented $(1,366,000$ in males and 396,000 in females) and 1,448,000 liver cancer deaths could be avoided $(1,079,000$ in males and 369,000 in females) from 2015 to 2030 in China.

\section{Discussion}

This is an updated systematic analysis of nationwide disease burden of liver cancer in China by using the latest representative data collected from 339 qualified cancer registries and projections to 2030 . It was estimated that about 364,800 liver cancer cases and 318,800 liver cancer deaths in China in 2014. Liver cancer ranked the 4th in cancer incidence and the 2nd in cancer death in China, which is different in developed countries with ranks of the 11 th and 7 th respectively (25). Both the incidence and mortality rates of liver cancer were higher in rural areas,

Table 3 Predicted number of new liver cancer cases and deaths in China and changes between 2014 and 2030 apportioned into changes because of risk and demographics by gender

\begin{tabular}{|c|c|c|c|c|c|c|c|c|c|}
\hline \multirow[b]{2}{*}{ Sex } & \multicolumn{4}{|c|}{ Number of new cases or deaths } & \multicolumn{5}{|c|}{ Changes between 2014 and 2030 (\%) } \\
\hline & 2014 & 2020 & 2025 & 2030 & $\begin{array}{c}\text { Total } \\
\text { Change }\end{array}$ & $\begin{array}{c}\text { Change due } \\
\text { to risk }\end{array}$ & $\begin{array}{l}\text { Change due to } \\
\text { demographic }\end{array}$ & $\begin{array}{l}\text { Change due to } \\
\text { age structure }\end{array}$ & $\begin{array}{l}\text { Change due to } \\
\text { population size }\end{array}$ \\
\hline \multicolumn{10}{|l|}{ Incidence } \\
\hline All & 364,800 & 357,800 & 324,900 & 295,800 & -18.90 & -64.56 & 45.67 & 40.67 & 5.00 \\
\hline Male & 268,900 & 257,800 & 227,900 & 200,300 & -25.52 & -66.46 & 40.94 & 36.49 & 4.45 \\
\hline Female & 95,900 & 100,000 & 97,000 & 95,600 & -0.32 & -57.99 & 57.67 & 52.10 & 5.57 \\
\hline \multicolumn{10}{|l|}{ Mortality } \\
\hline All & 318,800 & 315,900 & 298,200 & 280,700 & -11.95 & -60.99 & 49.04 & 44.05 & 5.00 \\
\hline Male & 233,500 & 227,600 & 211,200 & 194,500 & -16.70 & -60.59 & 43.88 & 39.43 & 4.45 \\
\hline Female & 85,300 & 88,300 & 87,000 & 86,200 & 1.09 & -60.75 & 61.84 & 56.27 & 5.57 \\
\hline
\end{tabular}


especially for those younger than 65 years, both in males and females. Notably, the less-developed western areas of China had the highest incidence and mortality rates, followed by middle and eastern areas. In the western areas, liver cancer ranked the 2 nd for both incidence and mortality, accounting for $11.88 \%$ of all cancer cases and $16 \%$ of all cancer deaths. While in the eastern areas, it ranked in the 5 th for incidence and the 2 nd for mortality, accounting for $8.02 \%$ of all cancer cases and $12.25 \%$ of all cancer deaths in 2014. The temporal trends in agestandardized incidence and mortality rates continued decreasing over the period of 2000 to 2014 and will decrease by about $44 \%$ between 2014 and 2030, but the number of new cases and deaths showed stable compared with previous publication (26) because of the increasing size of the population and population aging, with changing risk contributing about $-60 \%$, changing age structures contributing about $40 \%$. The hospitalization expenses of treatment for liver cancers in China increased from 4,930 million in 2011 to 81,400 million RMB in 2015 (27) and may decrease in the future with the decrease of new incident cases. Liver cancer has a poor prognosis, with a low 5 -year relative survival rate of about $12.1 \%$ in China (28), and forecasts could help to support prevention and treatment strategies to reduce the incidence and burden of liver cancer. Further analysis showed that the average incident age of liver cancer (being diagnosed) delayed about 3-5 years over the past 15 years as shown in Supplementary Table S3.

In China, chronic HBV infection has been identified as the dominant risk factor of liver cancer $(4,6)$. Neonatal hepatitis $\mathrm{B}$ vaccination proved efficiently in China to prevent HBV-related liver cancer (16). To control the disease, $\mathrm{HBV}$ vaccination to newborns was integrated into national immunization program since 1992 in China, but the family paid for the costs. The coverage of 3-dose HBV vaccination was nearly $97 \%$ in urban areas but only about $50 \%$ in rural areas by 1994 and $63 \%$ by 2001, even lower in the economically less-developed western areas of China. The Ministry of Public health of China then integrated the neonatal $\mathrm{HBV}$ vaccination into the nationwide immunization program since 2002 with the vaccines provided entirely by the government starting from 2002 $(18,29)$. Thus, one of the reasons of the highest liver cancer incidence currently in western areas might be due to the delayed vaccination coverage.

Occurrence of liver cancer mainly reflected the historical exposure to a variety of risk factors such as $\mathrm{HBV}$ infections, tobacco smoking or alcohol consumption. Multifaceted preventive intervention based on multiple etiologic factors might be the most promising approaches. $\mathrm{HBV}$ vaccination and the progress in the treatment of hepatitis B were the most effective approaches to reduce liver cancer incidence during the past decades, though the oldest generation receiving the neonatal $\mathrm{HBV}$ vaccination in China since 1992 was younger than 30 years in 2014. The trend for liver cancer decreases was mainly observed in the younger age groups ( $<50$ years), predominantly in those below 40 years during 2000-2014, which may partially reflect the effect of vaccination for $\mathrm{HBV}$, and the decline trend in age group of 40-49 years can reflect the protective effect on controlling the other factors, including the progress in the treatment and control of hepatitis B, safe food and clean water in China (7). A previous study (6) gives the contribution of different risk factors for liver cancer including ever-smoking, hepatitis B, hepatitis C, alcohol, excess body weight and other causes varies between regions and gender as shown in Figure 2. It was estimated that HBV contributed to about $59.3 \%$ of liver cancer in China, but only $8.7 \%$ can be attributed to HCV. The decreasing trends during the past decades and the predicted decreasing in the future can be considered as a comprehensive reflection of the change of risk factors.

Currently, there was an estimated about 93 million individuals infected with HBV (30). The experience in some rural areas of China by controlling the potential environmental carcinogens exposures might be practical strategies for reducing the liver cancer in younger people $(16,31)$. Improving the personal lifestyle by improving the dietary pattern may also reduce the liver cancer risk $(3,32,33)$. In addition, direct acting antiviral therapies by nucleos $(\mathrm{t})$ ides analogues for patients with chronic hepatitis B or C might be another option (3). The Chinese government has initiated some programs to control the $\mathrm{HBV}$ infection including $\mathrm{HBV}$ vaccination to neonates, and some antiviral nucleot(s)ides analogues for the patients with active chronic hepatitis B were covered by healthcare. The disease burden caused by liver cancer will be relieved gradually.

However, with the changing lifestyle in recent decades in China, chronic HCV infection, heavy alcohol consumption, cigarette smoking and overweight, which have been considered as important risk factors of liver cancer in developed countries, became evident in China (34). We noticed the decline of liver cancer incidence in 


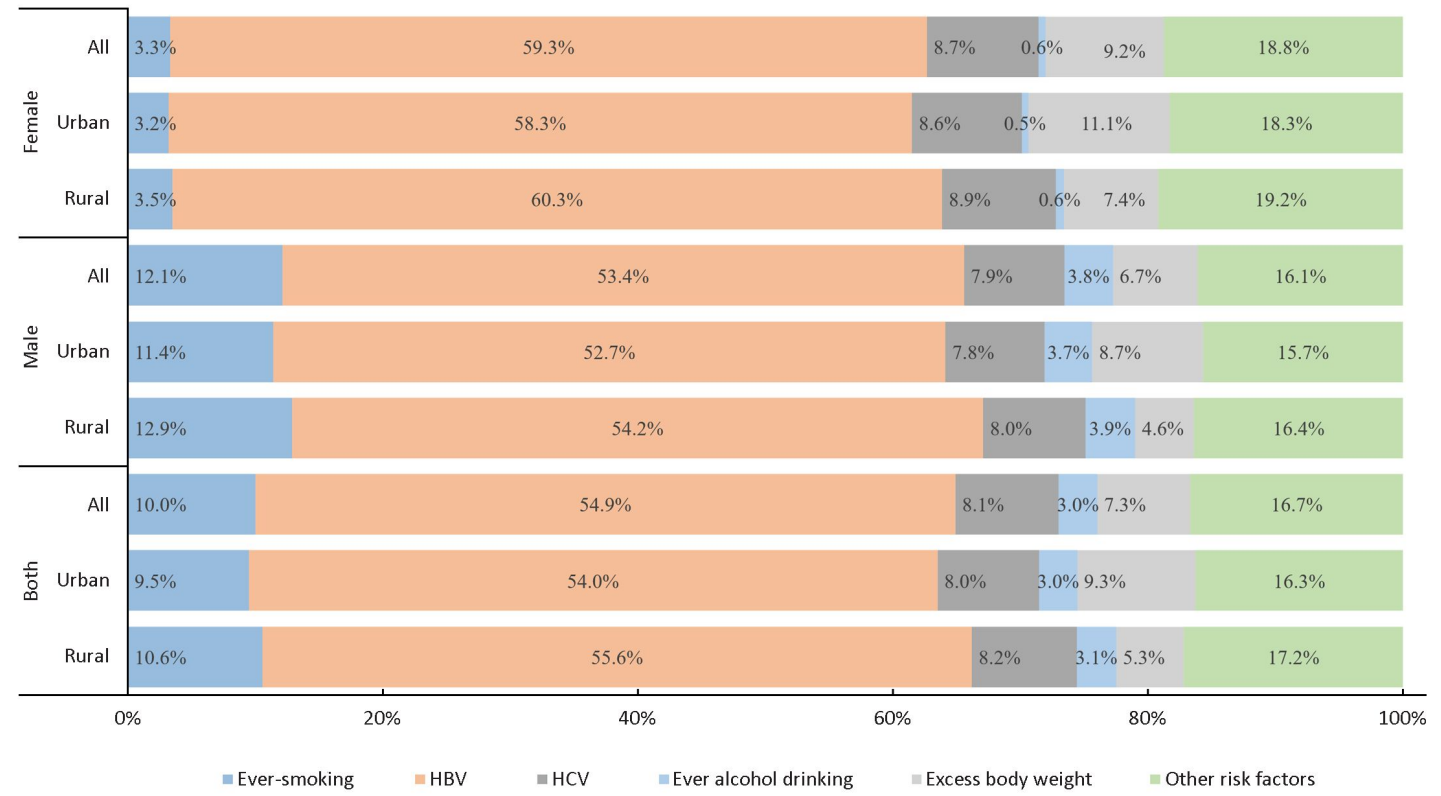

Figure 2 Contribution of ever-smoking, hepatitis B, hepatitis C, alcohol, excess body weight and other causes on liver cancer incidence both sexes and by areas in China.

the 50-59 age group of females and rural males, but not the urban males during 2000-2014. Therefore, these identified risk factors in developed countries should be well controlled in the future of China for reducing the liver cancer disease burden.

Predicting future cancer occurrence is filled with uncertainty but can help policymaker to redistribute the resources for liver cancer prevention in China. Highquality and long-term population-based data are prerequisites for making sensible predictions. The statistical model that used in prediction in this study is the age-period-cohort model, the most commonly used model in prediction in which the period and birth cohort effects could be considered as proxies for events such as risk factors for which we often cannot measure directly (35). Previous studies have shown the predicted trend for liver cancer in the future in China but limited to too few registries and the results are not reliable (36). In this study, the updated systematic statistics for liver cancer in China in 2014 was estimated based on 339 cancer registries covering about 288 million population and this is the most representative data that can be obtained so far. The temporal trends in incidence and mortality from 2000 to 2014 were based on the data from 22 high-quality cancer registries and the population forecasts made by China Population and Development Research Center. The small proportion covered by those 22 registries may hardly represent the nationwide population and may lead to some unknown levels of uncertainty for estimates, however, all those conditions above should make sensible predictions for liver cancer in the future in China.

The key strength of this study is the use of the most representative data from NCC, but the predictions give the assumption that trends observed in the past would continue in the future. However, the younger generation showed a more obvious decreasing trend, and this may demonstrate that the younger the population, the more they can benefit from the vaccine or other precautions, which consequently might cause the falling liver cancer burden accelerating in the future. Due to the lack of effective treatment options, these results highlight the need for ongoing prevention and control strategies to reduce the burden of liver cancer, and more efforts should especially focus on younger generations.

\section{Acknowledgements}

We express deep gratitude to all the local Cancer Registry staff in China for contributing to the data collection, validation, and routine analyses. This study was supported by National Natural Science Foundation of China (No. 81602931), Ministry of Science and Technology (No. 2014FY121100) and State Key Projects Specialized on Infectious Diseases (No. 2012ZX10002008). 


\section{Footnote}

Conflicts of Interest: The authors have no conflicts of interest to declare.

\section{References}

1. Bray F, Ferlay J, Soerjomataram I, et al. Global cancer statistics 2018: GLOBOCAN estimates of incidence and mortality worldwide for 36 cancers in 185 countries. CA Cancer J Clin 2018;68:394-424.

2. Sia D, Villanueva A, Friedman SL, et al. Liver cancer cell of origin, molecular class, and effects on patient prognosis. Gastroenterology 2017;152:745-61.

3. Massarweh NN, El-Serag HB. Epidemiology of hepatocellular carcinoma and intrahepatic cholangiocarcinoma. Cancer Control 2017;24:1073274817 729245 .

4. de Martel C, Maucort-Boulch D, Plummer M, et al. World-wide relative contribution of hepatitis $\mathrm{B}$ and $\mathrm{C}$ viruses in hepatocellular carcinoma. Hepatology 2015;62:1190-200.

5. Poon D, Anderson BO, Chen LT, et al. Management of hepatocellular carcinoma in Asia: consensus statement from the Asian Oncology Summit 2009. Lancet Oncol 2009;10:1111-8.

6. Islami F, Chen $\mathrm{W}, \mathrm{Yu} \mathrm{XQ}$, et al. Cancer deaths and cases attributable to lifestyle factors and infections in China, 2013. Ann Oncol 2017;28:2567-74.

7. Sun Z, Chen T, Thorgeirsson SS, et al. Dramatic reduction of liver cancer incidence in young adults: 28 year follow-up of etiological interventions in an endemic area of China. Carcinogenesis 2013;34: 1800-5.

8. Papatheodoridis GV, Idilman R, Dalekos GN, et al. The risk of hepatocellular carcinoma decreasing after the first 5 years of entecavir or tenofovir in Caucasians with chronic hepatitis B. Hepatology 2017;66: 1444-53.

9. Wu CY, Lin JT, Ho HJ, et al. Association of nucleos(t)ide analogue therapy with reduced risk of hepatocellular carcinoma in patients with chronic hepatitis B: a nationwide cohort study. Gastroenterology 2014;147:143-51. e5.

10. Islami F, Miller KD, Siegel RL, et al. Disparities in liver cancer occurrence in the United States by race/ethnicity and state. CA Cancer J Clin
2017;67:273-89.

11. Global Burden of Disease Liver Cancer Collaboration. The Burden of Primary Liver Cancer and Underlying Etiologies From 1990 to 2015 at the Global, Regional, and National Level: Results From the Global Burden of Disease Study 2015. JAMA Oncol 2017;3:1683-91.

12. White DL, Thrift AP, Kanwal F, et al. Incidence of hepatocellular carcinoma in all 50 United States, from 2000 through 2012. Gastroenterology 2017;152:81220. e5.

13. Wang M, Wang Y, Feng X, et al. Contribution of hepatitis $\mathrm{B}$ virus and hepatitis $\mathrm{C}$ virus to liver cancer in China north areas: Experience from Chinese National Cancer Center. Int J Infect Dis 2017;65:1521.

14. Wu M, Shen F. Liver Cancer. 3rd ed. Beijing: Peking University Medical Press, 2010:327.

15. Chiang CJ, Yang YW, You SL, et al. Thirty-year outcomes of the national hepatitis B immunization program in Taiwan. JAMA 2013;310:974-6.

16. Qu C, Chen T, Fan C, et al. Efficacy of neonatal $\mathrm{HBV}$ vaccination on liver cancer and other liver diseases over 30-year follow-up of the Qidong hepatitis B intervention study: a cluster randomized controlled trial. PLoS Med 2014;11:e1001774.

17. Chen $W$, Zheng R, Baade PD, et al. Cancer statistics in China, 2015. CA Cancer J Clin 2016;66:115-32.

18. Qu C, Duan Z, Chen K, et al. Reducing liver cancer risk beginning at birth: experiences of preventing chronic hepatitis $\mathrm{B}$ virus infection in China. Hepatoma Res 2017;3:228-40.

19. Torre LA, Bray F, Siegel RL, et al. Global cancer statistics, 2012. CA Cancer J Clin 2015;65:87-108.

20. Ferlay J, Soerjomataram I, Ervik $M$, et al. GLOBOCAN 2012 v1.0, Cancer Incidence and Mortality Worldwide: IARC CancerBase No. 11. International Agency for Research on Cancer, 2013. Available online: http://globocan.iarc.fr

21. Allemani C, Weir HK, Carreira H, et al. Global surveillance of cancer survival 1995-2009: analysis of individual data for 25,676,887 patients from 279 population-based registries in 67 countries (CONCORD-2). Lancet 2015;385:977-1010.

22. Bray F, Guilloux A, Sankila R, et al. Practical implications of imposing a new world standard 
population. Cancer Causes Control 2002;13:175-82.

23. Malvezzi M, Carioli G, Bertuccio P, et al. European cancer mortality predictions for the year 2017, with focus on lung cancer. Ann Oncol 2017;28:1117-23.

24. Møller B, Fekjaer H, Hakulinen T, et al. Prediction of cancer incidence in the Nordic countries up to the year 2020. Eur J Cancer Prev 2002;11 Suppl 1:S1-96.

25. Global Burden of Disease Cancer Collaboration. The global burden of cancer 2013. JAMA Oncol 2015;1:505-27.

26. Chen $\mathrm{W}$, Zheng $\mathrm{R}$, Zhang S, et al. Cancer incidence and mortality in China, 2013. Cancer Lett 2017; 401:63-71.

27. Cai Y, Xue M, Chen W, et al. Expenditure of hospital care on cancer in China, from 2011 to 2015. Chin J Cancer Res 2017;29:253-62.

28. Zeng H, Chen W, Zheng R, et al. Changing cancer survival in China during 2003-15: a pooled analysis of 17 population-based cancer registries. Lancet Glob Health 2018;6:e555-e567.

29. Sun Z, Ming L, Zhu X, et al. Prevention and control of hepatitis B in China. J Med Virol 2002;67:447-50.

30. Liu J, Zhang S, Wang Q, et al. Seroepidemiology of hepatitis $B$ virus infection in 2 million men aged 21-

Cite this article as: Zheng $\mathrm{R}, \mathrm{Qu} \mathrm{C}$, Zhang S, Zeng H, Sun K, Gu X, Xia C, Yang Z, Li H, Wei W, Chen W, He J. Liver cancer incidence and mortality in China: Temporal trends and projections to 2030. Chin J Cancer Res 2018;30(6):571579. doi: 10.21147/j.issn.1000-9604.2018.06.01
49 years in rural China: a population-based, crosssectional study. Lancet Infect Dis 2016;16:80-6.

31. Chen JG, Egner PA, Ng D, et al. Reduced aflatoxin exposure presages decline in liver cancer mortality in an endemic region of China. Cancer Prev Res (Phila) 2013;6:1038-45.

32. Zhang $W$, Shu XO, Li H, et al. Vitamin intake and liver cancer risk: a report from two cohort studies in China. J Natl Cancer Inst 2012;104:1173-81.

33. Kurahashi N, Inoue M, Iwasaki M, et al. Vegetable, fruit and antioxidant nutrient consumption and subsequent risk of hepatocellular carcinoma: a prospective cohort study in Japan. Br J Cancer 2009; 100:181-4.

34. Wang FS, Fan JG, Zhang Z, et al. The global burden of liver disease: the major impact of China. Hepatology 2014;60:2099-108.

35. Bray F, Møller B. Predicting the future burden of cancer. Nat Rev Cancer 2006;6:63-74.

36. Wu J, Yang S, Xu K, et al. Patterns and trends of liver cancer incidence rates in Eastern and Southeastern Asian countries (1983-2007) and predictions to 2030. Gastroenterology 2018;154:1719-28. e5. 


\section{Supplementary materials}

The National Central Cancer Registry of China (NCCRC) is responsible for collecting data from local population-based cancer registries and evaluating and publishing national cancer statistics of China. Every cancer registry collects records of cancer patients from hospitals, community health service centers, the death surveillance system and the Basic Medical Insurances for Urban Residents and the New-Rural Cooperative Medical System. The information for each new cancer patient was collected including personal identification number, age at diagnosis, gender, date of diagnosis, cancer site, and basis of diagnosis, and so on. By 1 June 2017, 449 cancer registries from all 31 provinces submitted incidence and mortality data of 2014 to NCCRC, which covered 345,711,646 population, accounting for about $25.27 \%$ of the national population. All cancer cases were coded according to the International Classification of Diseases for Oncology, 3rd edition (ICD-O-3) and the International Statistical Classification of Diseases and Related Health Problems 10th Revision (ICD-10).

All data submitted to NCCRC were under quality control based on the criteria of "Guideline for Chinese Cancer Registration" and "Cancer Incidence in Five Continents Volume IX" by International Agency for Research on Cancer (IARC)/International Association of Cancer Registries (IACR). Briefly, the completeness, comparability and validity of the data were evaluated using a series of standards including the mortality to incidence (M/I) ratio, the percentage of cases morphologically verified (MV\%), the percentage of death certificate-only cases (DCO\%), and stability of cancer statistics over years. Questionable records were returned to the local registries for data check and correction. Data classified as category A or B were deemed acceptable for inclusion in the study. After quality control, 339 registries' data from 31 provinces which met the criteria were included in this analysis. The detailed distribution of the 339 cancer registries were shown in Supplementary Figure S1. Of the 339 cancer registries, 129 were located in urban areas (covered 144,061,915 population) and 210 were in rural areas (covered 144,181,432 population). The overall population covered about 288,243,347 population (146,203,891 males and 142,039,456 females), accounting for 21.07\% of national population in China in 2014.

Crude incidence and mortality rates of liver cancers were calculated by area (urban/rural), gender (male/female) and age groups (0, 1-4, 5 to 80 by 5 years, $85+)$ using the 339 cancer registries' data. We estimated the numbers of new cancer cases and deaths in China in 2014 by applying age-specific incidence rates from 339 cancer registries in 2014 to the national agespecific population. The age-specific incidence rates from 339 cancer registries multiplied by the corresponding estimated age-specific population in each stratum and added them up to calculate the estimated numbers of new cases of cancer incidence and deaths. The national population data by five-year age group and sex were obtained from statistics or public security census (http://data.stats.gov.cn/), with about 1,367.82 million population in 2014 in China (749.16 million population in urban areas and 618.66 million population in rural areas). Each cancer registry provided population data in the respective areas to the NCCRC. These data were sourced from local Statistical or Public Security Bureaus, or from calculations based on census data. The world standard population (Segi's population) was used for age-standardized rates.

Temporal trends in incidence and mortality from 2000 to 2014 were examined by fitting joinpoint models (https://surveillance.cancer.gov/joinpoint/) to the age-standardized rates (per 100,000 population) using data from 22 cancer registries of China. Those 22 cancer registries were established for more than 15 years and represented overall $3.34 \%$ of the Chinese population, standardized according to the world standard population. To reduce the possibility of reporting spurious changes in trends over the period, all models were restricted to a maximum of 2 joinpoints ( 3 line segments). Trends were expressed as an annual percentage change (APC) and the $Z$ test was used to assess whether the APC was statistically different from zero. In describing trends, the terms "increase" or "decrease" were used when the slope (APC) of the trend was statistically significant $(\mathrm{P}<0.05)$. For non-statistically significant trends, the term "stable" were used.

\section{Etiology splits}

To find the proportion of liver cancer cases due to the six etiology groups included ever-smoking, hepatitis B, hepatitis C, alcohol, excess body weight and other causes. Data on tobacco smoking, past-year alcohol drinking, body mass indexes (BMI) were obtained from the China National Nutrition and Health Survey 2002 (1). Relative risks for the association between 
ever-smoking, or ever-alcohol drinking and cancer were mostly obtained from prospective studies in China or from metaanalyses that had examined the associations in China or Asia, and when these were not available, from other sources. Relative risks for overweight were obtained from meta-analyses of studies worldwide because relative risks from large-scale studies in China were scarce. Relative risks for infections and cancer were mostly obtained from an analysis of global cancer burden of infections $(2,3)$. The result has been described in detail elsewhere (4).

\section{References}

1. Wu Y, Huxley R, Li L, et al. Prevalence, awareness, treatment, and control of hypertension in China: data from the China National Nutrition and Health Survey 2002. Circulation 2008;118:2679-86.

2. de Martel C, Ferlay J, Franceschi S, et al. Global burden of cancers attributable to infections in 2008: a review and synthetic analysis. Lancet Oncol 2012;13:607-15.

3. Plummer M, de Martel C, Vignat J, et al. Global burden of cancers attributable to infections in 2012: a synthetic analysis. Lancet Glob Health 2016;4:e609-16.

4. Islami F, Chen W, Yu XQ, et al. Cancer deaths and cases attributable to lifestyle factors and infections in China, 2013. Ann Oncol 2017;28:2567-74. 


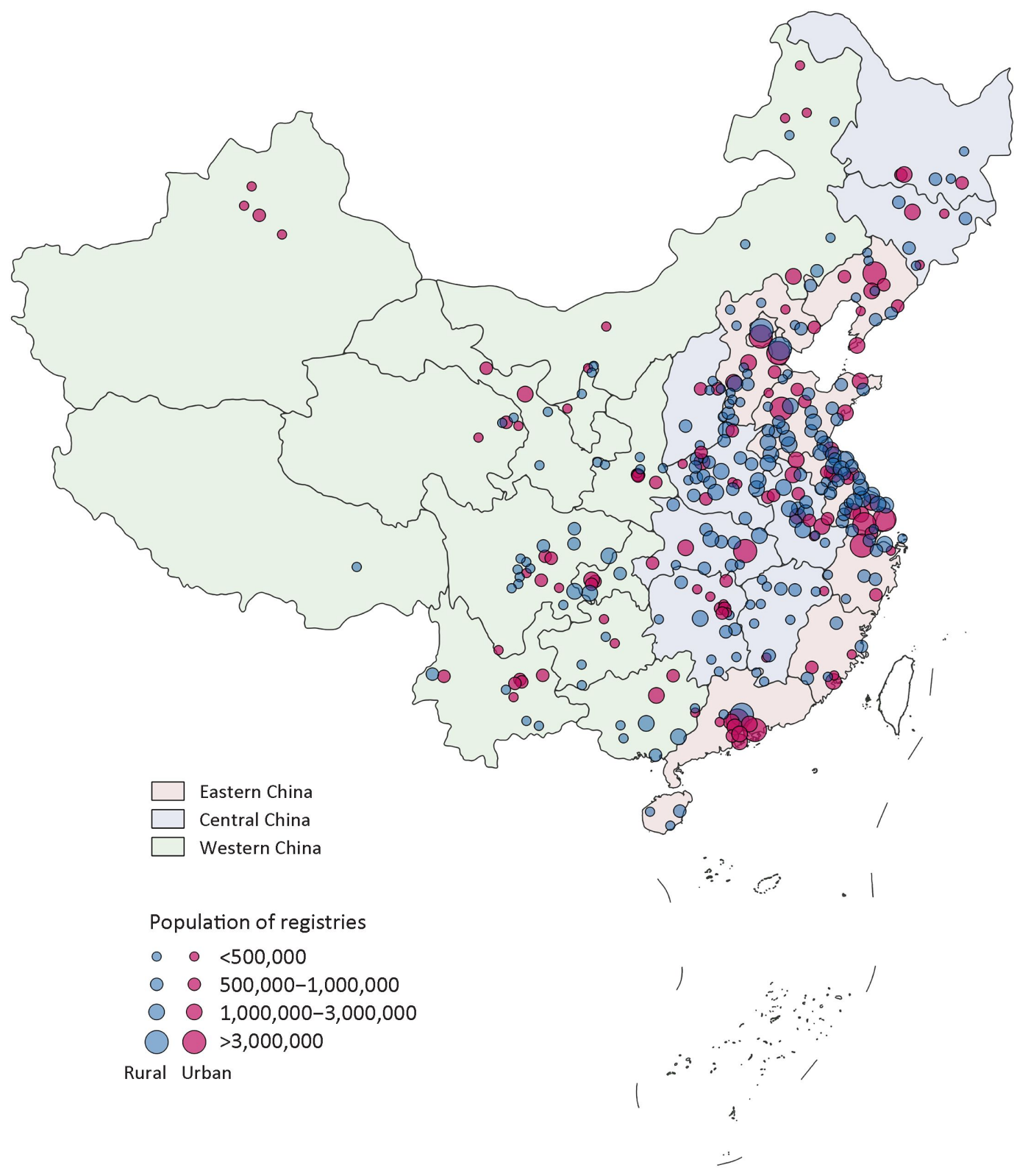

Figure S1 Distribution of 339 selected cancer registries in China. 

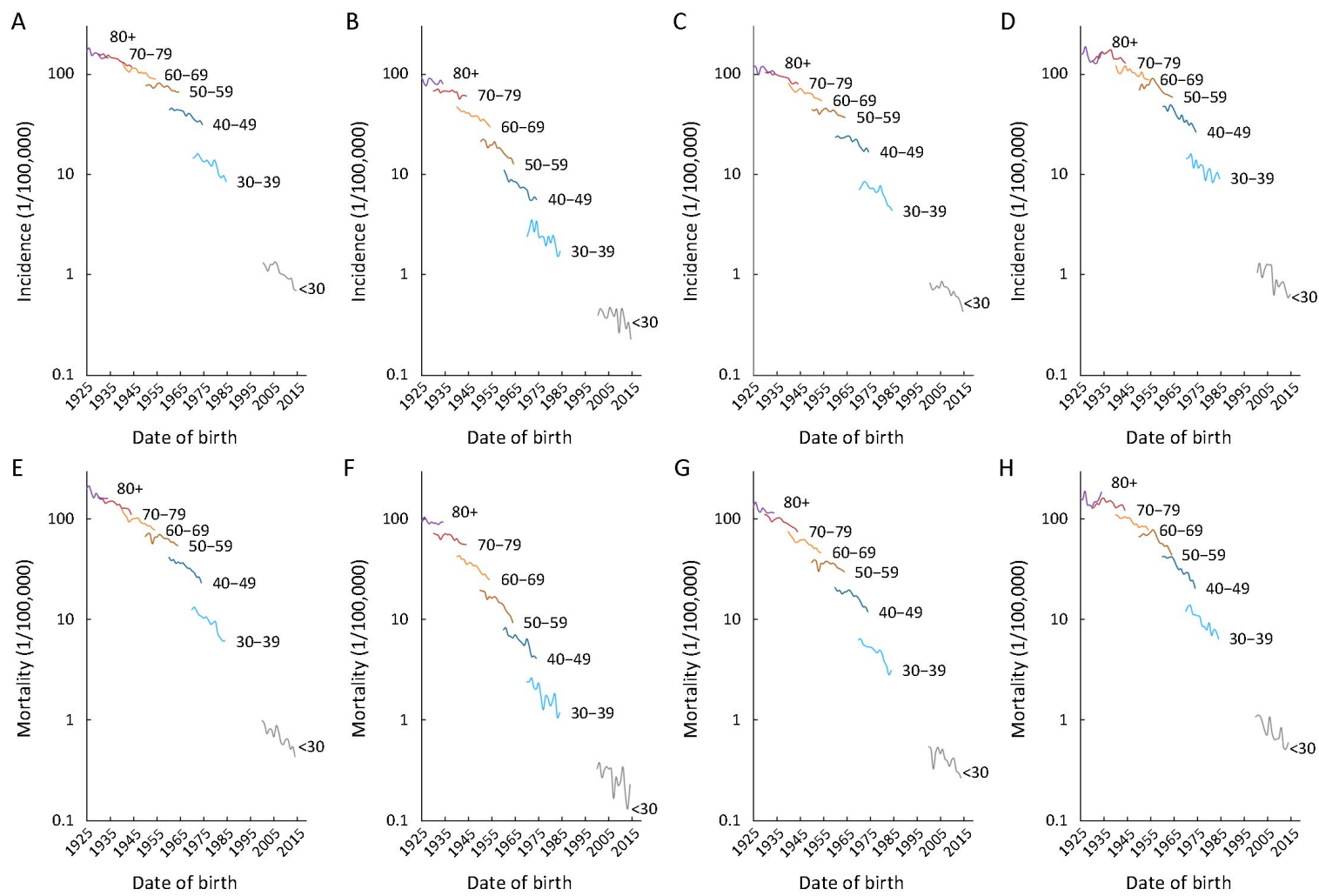

Figure S2 Liver cancer incidence and mortality rates with different birth cohorts by age groups in China. (A) Male incidence; (B) Female incidence; (C) Urban incidence; (D) Rural incidence; (E) Male mortality; (F) Female mortality; (G) Urban mortality; (H) Rural mortality.

Table S1 Liver cancer incidence trends by areas, gender and age, 2000 to 2014

\begin{tabular}{|c|c|c|c|c|c|c|c|c|c|}
\hline \multirow{2}{*}{ Areas } & \multirow{2}{*}{ Sex } & \multirow{2}{*}{ Age (year) } & \multicolumn{2}{|c|}{ Trend 1} & \multicolumn{2}{|c|}{ Trend 2} & \multicolumn{2}{|c|}{ Trend 3} & \multirow{2}{*}{$\begin{array}{c}\text { AAPC } \\
2000-2014\end{array}$} \\
\hline & & & Period & APC & Period & APC & $2000-2014$ & APC & \\
\hline All areas & Both sexes & ASIR & $2000-2006$ & -0.9 & $2006-2014$ & $-3.0^{*}$ & & & $-2.2^{*}$ \\
\hline All areas & Both sexes & $0-39$ & 2000-2002 & 4.9 & 2002-2014 & $-4.5^{\star}$ & & & $-3.9^{*}$ \\
\hline All areas & Both sexes & $40-49$ & 2000-2014 & $-3.0^{*}$ & & & & & $-3.0^{*}$ \\
\hline All areas & Both sexes & $50-59$ & 2000-2006 & 0.2 & 2006-2014 & $-2.8^{*}$ & & & $-1.6^{*}$ \\
\hline All areas & Both sexes & $60-69$ & 2000-2002 & $-8.3^{*}$ & 2002-2005 & 1.3 & 2005-2014 & $-2.7^{\star}$ & $-2.2^{*}$ \\
\hline All areas & Both sexes & $70-79$ & 2000-2007 & -0.9 & 2007-2014 & $-2.7^{\star}$ & & & $-1.8^{*}$ \\
\hline All areas & Both sexes & $80+$ & 2000-2014 & -0.6 & & & & & -0.6 \\
\hline All areas & Male & ASIR & $2000-2006$ & -0.9 & 2006-2014 & $-3.0^{*}$ & & & $-2.1^{*}$ \\
\hline All areas & Male & $0-39$ & $2000-2009$ & $-2.5^{\star}$ & 2009-2014 & $-6.8^{\star}$ & & & $-3.9^{*}$ \\
\hline All areas & Male & $40-49$ & 2000-2005 & -0.6 & 2005-2014 & $-3.4^{\star}$ & & & $-2.6^{*}$ \\
\hline All areas & Male & $50-59$ & 2000-2006 & 0.7 & 2006-2014 & $-2.3^{\star}$ & & & $-1.1^{*}$ \\
\hline All areas & Male & $60-69$ & 2000-2002 & $-9.8^{*}$ & 2002-2005 & 2.1 & 2005-2014 & $-2.8^{*}$ & $-2.2^{*}$ \\
\hline All areas & Male & $70-79$ & 2000-2007 & $-1.5^{*}$ & 2007-2014 & $-3.0^{*}$ & & & $-2.2^{*}$ \\
\hline All areas & Male & $80+$ & 2000-2014 & $-1.9^{*}$ & & & & & $-1.9^{*}$ \\
\hline All areas & Female & ASIR & 2000-2008 & $-1.6^{*}$ & 2008-2014 & $-3.7^{*}$ & & & $-2.4^{*}$ \\
\hline All areas & Female & $0-39$ & 2000-2014 & $-3.4^{*}$ & & & & & $-3.4^{*}$ \\
\hline All areas & Female & $40-49$ & 2000-2014 & $-4.4^{\star}$ & & & & & $-4.4^{*}$ \\
\hline All areas & Female & $50-59$ & 2000-2006 & -1.2 & 2006-2014 & $-4.9^{*}$ & & & $-3.5^{\star}$ \\
\hline
\end{tabular}

Table S1 (continued) 
Table S1 (continued)

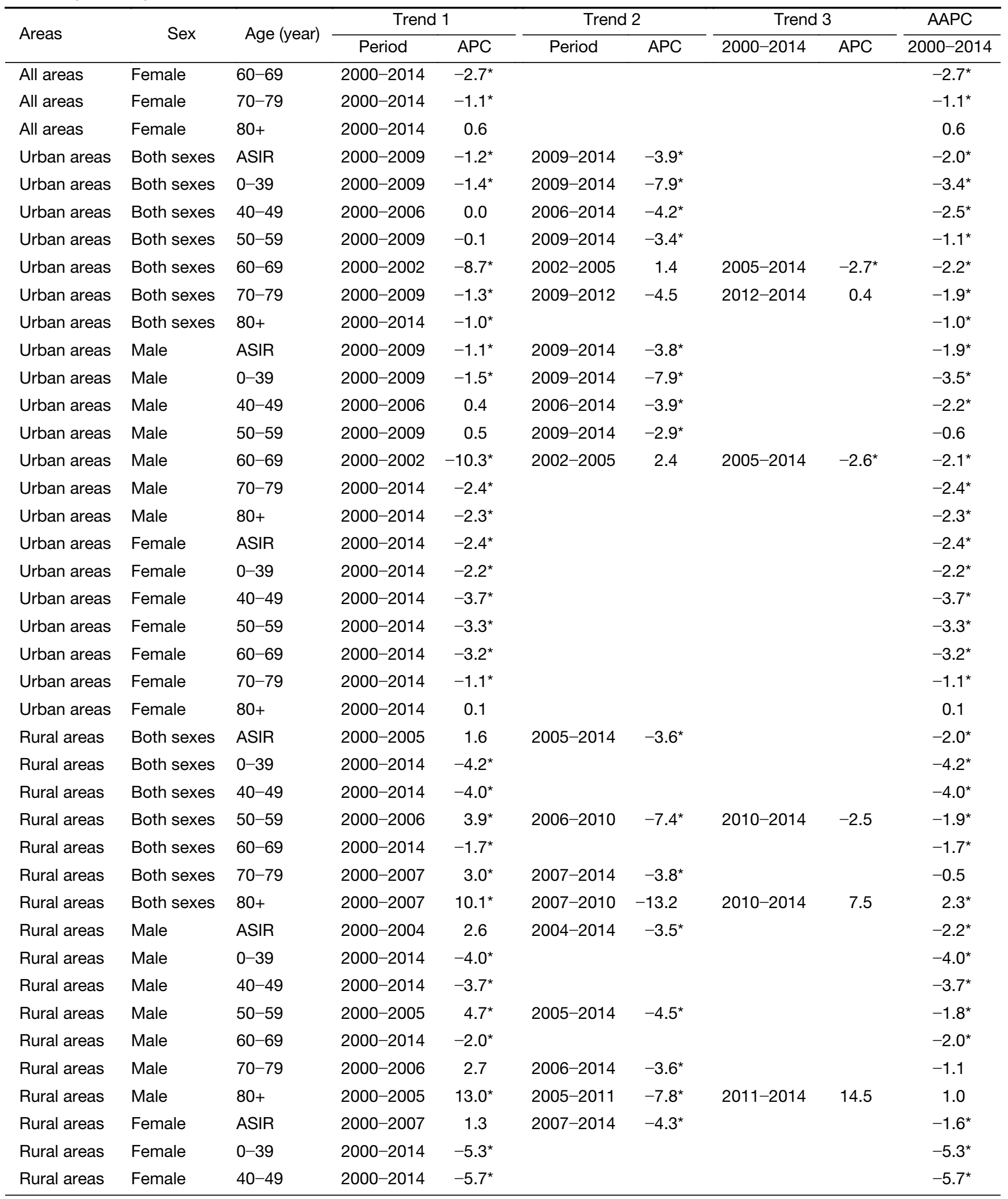

Table S1 (continued) 
Table S1 (continued)

\begin{tabular}{|c|c|c|c|c|c|c|c|c|c|}
\hline \multirow{2}{*}{ Areas } & \multirow{2}{*}{ Sex } & \multirow{2}{*}{ Age (year) } & \multicolumn{2}{|c|}{ Trend 1} & \multicolumn{2}{|c|}{ Trend 2} & \multicolumn{2}{|c|}{ Trend 3} & \multirow{2}{*}{$\frac{\text { AAPC }}{2000-2014}$} \\
\hline & & & Period & APC & Period & APC & $2000-2014$ & APC & \\
\hline Rural areas & Female & $50-59$ & $2000-2006$ & $3.6^{*}$ & $2006-2014$ & $-5.6^{\star}$ & & & $-2.1^{*}$ \\
\hline Rural areas & Female & $70-79$ & 2000-2007 & $4.4^{*}$ & 2007-2014 & $-4.0^{*}$ & & & 0.1 \\
\hline Rural areas & Female & $80+$ & 2000-2007 & $12.3^{\star}$ & $2007-2010$ & -11.0 & 2010-2014 & 3.8 & $3.3^{*}$ \\
\hline
\end{tabular}

APC, annual percent change; AAPC, average annual percent change; ASIR, age-standardized incidence rate using world Segi's standard population; *, The APC or AAPC is significantly different from zero $(\mathrm{P}<0.05)$.

Table S2 Liver cancer mortality trends by areas, gender and age, 2000 to 2014

\begin{tabular}{|c|c|c|c|c|c|c|c|c|c|}
\hline \multirow{2}{*}{ Areas } & \multirow{2}{*}{ Sex } & \multirow{2}{*}{ Age (year) } & \multicolumn{2}{|c|}{ Trend 1} & \multicolumn{2}{|c|}{ Trend 2} & \multicolumn{2}{|c|}{ Trend 3} & \multirow{2}{*}{$\frac{\text { AAPC }}{2000-2014}$} \\
\hline & & & Period & APC & Period & $\mathrm{APC}$ & Period & APC & \\
\hline All areas & Both sexes & ASMR & $2000-2003$ & $-5.1^{*}$ & $2003-2006$ & 1.5 & $2006-2014$ & $-3.9^{\star}$ & $-2.6^{\star}$ \\
\hline All areas & Both sexes & $40-49$ & 2000-2010 & $-2.8^{\star}$ & 2010-2014 & $-7.4^{\star}$ & & & $-3.8^{*}$ \\
\hline All areas & Both sexes & $50-59$ & 2000-2014 & $-2.0^{\star}$ & & & & & $-2.0^{\star}$ \\
\hline All areas & Both sexes & $70-79$ & 2000-2003 & $-3.7^{\star}$ & 2003-2006 & 1.8 & 2006-2014 & $-3.4^{\star}$ & $-2.1^{\star}$ \\
\hline All areas & Both sexes & $80+$ & 2000-2014 & $-0.7^{\star}$ & & & & & $-0.7^{\star}$ \\
\hline All areas & Male & ASMR & 2000-2003 & $-5.4^{*}$ & 2003-2006 & 1.7 & 2006-2014 & $-3.7^{\star}$ & $-2.6^{\star}$ \\
\hline All areas & Male & $0-39$ & 2000-2014 & $-5.1^{*}$ & & & & & $-5.1^{*}$ \\
\hline All areas & Male & $40-49$ & 2000-2009 & $-2.5^{\star}$ & 2009-2014 & $-6.1^{*}$ & & & $-3.6^{\star}$ \\
\hline All areas & Male & $80+$ & 2000-2014 & $-1.8^{*}$ & & & & & $-1.8^{*}$ \\
\hline All areas & Female & ASMR & 2000-2003 & $-4.5^{\star}$ & 2003-2006 & 0.7 & 2006-2014 & $-4.3^{\star}$ & $-3.1^{*}$ \\
\hline All areas & Female & $0-39$ & 2000-2014 & $-4.7^{\star}$ & & & & & $-4.7^{\star}$ \\
\hline All areas & Female & $40-49$ & 2000-2014 & $-4.4^{*}$ & & & & & $-4.4^{*}$ \\
\hline All areas & Female & $50-59$ & 2000-2009 & $-2.9^{\star}$ & 2009-2014 & $-8.3^{*}$ & & & $-4.5^{\star}$ \\
\hline All areas & Female & $60-69$ & 2000-2014 & $-3.7^{\star}$ & & & & & $-3.7^{\star}$ \\
\hline All areas & Female & $70-79$ & 2000-2003 & -3.7 & 2003-2006 & 3.2 & 2006-2014 & $-3.4^{*}$ & $-1.7^{\star}$ \\
\hline All areas & Female & $80+$ & 2000-2014 & 0.0 & & & & & 0.0 \\
\hline Urban areas & Both sexes & $80+$ & 2000-2014 & $-1.2^{*}$ & & & & & $-1.2^{*}$ \\
\hline Urban areas & Male & ASMR & 2000-2003 & $-6.3^{*}$ & 2003-2006 & 3.4 & 2006-2014 & $-3.8^{*}$ & $-2.3^{*}$ \\
\hline Urban areas & Male & $0-39$ & 2000-2014 & $-4.7^{\star}$ & & & & & $-4.7^{*}$ \\
\hline Urban areas & Male & $40-49$ & 2000-2003 & -3.7 & 2003-2006 & 2.7 & 2006-2014 & $-5.6^{\star}$ & $-3.2^{\star}$ \\
\hline Urban areas & Male & $50-59$ & 2000-2014 & -0.6 & & & & & -0.6 \\
\hline
\end{tabular}

Table S2 (continued) 
Table S2 (continued)

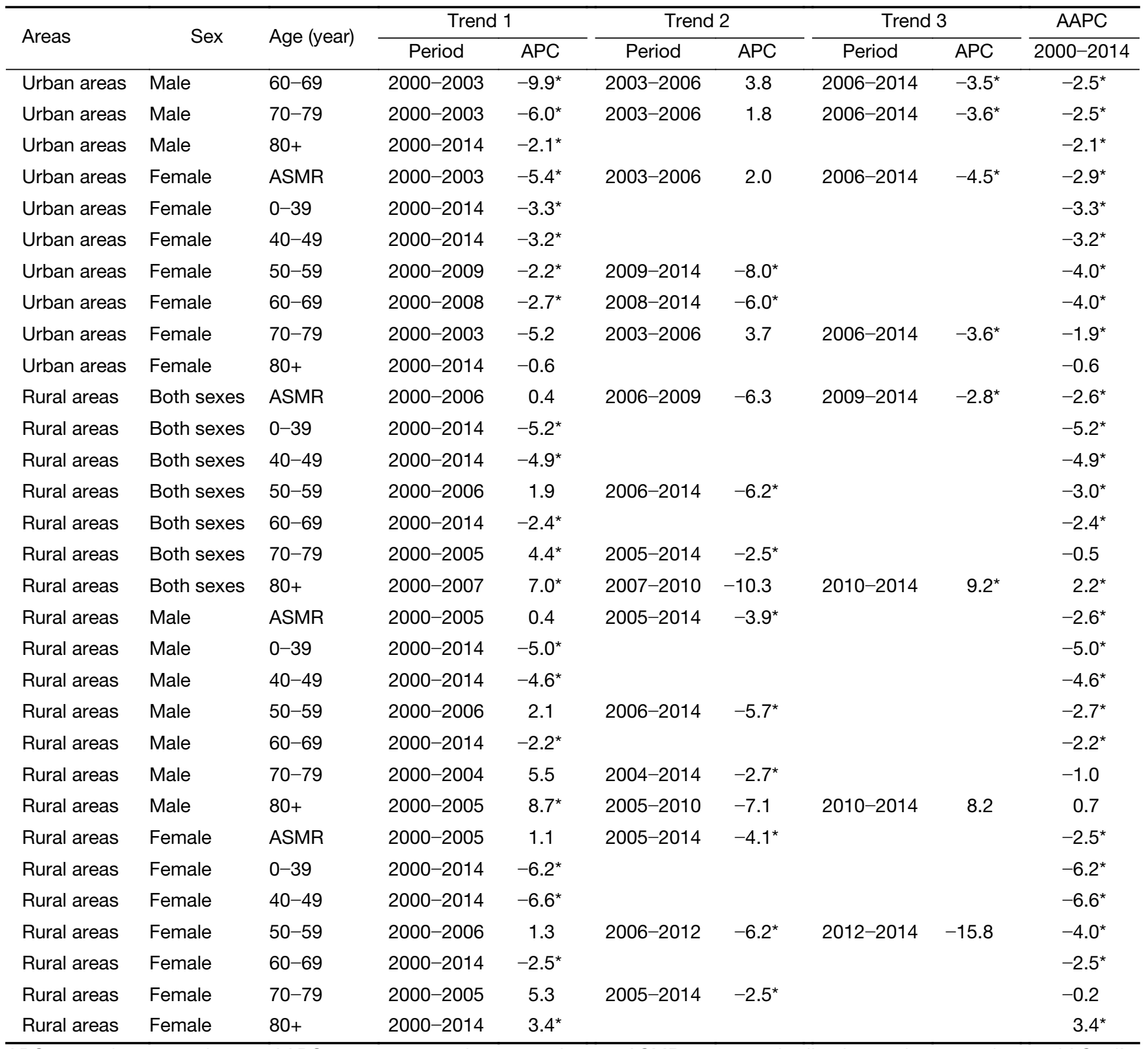

APC, annual percent change; AAPC, average annual percent change; ASMR, age-standardized mortaity rate using world Segi's standard population; " , The APC or AAPC is significantly different from zero $(\mathrm{P}<0.05)$. 
Table S3 Mean age of diagnosis of liver cancer, by gender and areas in 22 cancer registries

\begin{tabular}{|c|c|c|c|c|c|c|c|}
\hline \multirow{2}{*}{ Period } & \multirow{2}{*}{ Year } & \multicolumn{2}{|c|}{22 registries } & \multicolumn{2}{|c|}{ Urban } & \multicolumn{2}{|c|}{ Rural } \\
\hline & & Male & Female & Male & Female & Male & Female \\
\hline \multirow[t]{3}{*}{$2000-04$} & 2000 & 58.80 & 64.02 & 59.67 & 65.35 & 56.53 & 60.60 \\
\hline & 2004 & 59.35 & 64.66 & 59.82 & 65.50 & 57.83 & 62.57 \\
\hline & Changing & 0.55 & 0.64 & 0.15 & 0.15 & 1.30 & 1.97 \\
\hline \multirow[t]{3}{*}{ 2005-09 } & 2005 & 59.40 & 65.66 & 59.78 & 66.47 & 57.98 & 63.07 \\
\hline & 2009 & 60.40 & 67.00 & 60.66 & 67.78 & 59.31 & 64.33 \\
\hline & Changing & 1.00 & 1.34 & 0.88 & 1.31 & 1.33 & 1.26 \\
\hline \multirow[t]{3}{*}{ 2010-14 } & 2010 & 60.88 & 67.33 & 61.39 & 67.97 & 58.96 & 65.19 \\
\hline & 2014 & 62.35 & 68.99 & 62.66 & 69.87 & 61.20 & 66.07 \\
\hline & Changing & 1.47 & 1.66 & 1.27 & 1.90 & 2.24 & 0.88 \\
\hline \multirow[t]{5}{*}{$2000-14$} & 2000 & 58.80 & 64.02 & 59.67 & 65.35 & 56.53 & 60.60 \\
\hline & 2014 & 62.35 & 68.99 & 62.66 & 69.87 & 61.20 & 66.07 \\
\hline & Changing & 3.55 & 4.97 & 2.99 & 4.52 & 4.67 & 5.47 \\
\hline & $t$ & 18.70 & 20.50 & 11.52 & 11.69 & 17.35 & 11.14 \\
\hline & $P$ & $<0.001$ & $<0.001$ & $<0.001$ & $<0.001$ & $<0.001$ & $<0.001$ \\
\hline
\end{tabular}

